\title{
Vascular Inflammation Stratified by C-Reactive Protein and Low-Density Lipoprotein Cholesterol Levels: Analysis with ${ }^{18}$ F-FDG PET
}

\author{
Hye Jin Yoo*1, Sungeun Kim², Man Sik Park ${ }^{3}$, Sae Jeong Yang ${ }^{1}$, Tae Nyun Kim ${ }^{4}$, Ji A. Seo ${ }^{1}$, Sin Gon Kim ${ }^{1}$, \\ Nan Hee Kim ${ }^{1}$, Hong Seog Seo ${ }^{5}$, Sei Hyun Baik ${ }^{1}$, Dong Seop Choi ${ }^{1}$, and Kyung Mook Choi ${ }^{1}$ \\ ${ }^{I}$ Division of Endocrinology and Metabolism, Department of Internal Medicine, Korea University College of Medicine, Seoul, Korea; \\ ${ }^{2}$ Department of Nuclear Medicine, Korea University College of Medicine, Seoul, Korea; ${ }^{3}$ Department of Statistics, Sungshin Women's \\ University College of Natural Sciences, Seoul, Korea; ${ }^{4}$ Division of Endocrinology and Metabolism, Department of Internal Medicine, \\ College of Medicine, Inje University, Busan, Korea; and ${ }^{5}$ Cardiovascular Center, Korea University, Guro Hospital, Seoul, Korea
}

\begin{abstract}
We examined the severity of vascular inflammation in healthy individuals without hyperlipidemia but with elevated high-sensitivity C-reactive protein (hsCRP) using ${ }^{18} \mathrm{~F}-\mathrm{FDG}$ PET, which is a promising imaging technique for the assessment of vascular inflammation within atherosclerotic plaques. Methods: Vascular inflammation in the carotid arterial wall, represented as the targetto-background ratio (TBR), was measured using ${ }^{18} \mathrm{~F}-\mathrm{FDG}$ PET in 120 healthy subjects without a history of cardiovascular diseases. Results: Subjects with high hsCRP ( $\geq 2 \mathrm{mg} / \mathrm{L})$ and low low-density lipoprotein cholesterol (LDL-C) $(<130 \mathrm{mg} / \mathrm{dL})$ levels had a significantly higher maximum TBR than did those with low hsCRP $(<2 \mathrm{mg} / \mathrm{L})$ and low LDL-C levels $(<130 \mathrm{mg} / \mathrm{dL})$ or low $\mathrm{hsCRP}(<2$ $\mathrm{mg} / \mathrm{L})$ and high LDL-C levels ( $\geq 130 \mathrm{mg} / \mathrm{dL})(1.29 \pm 0.13,1.12 \pm$ 0.10 , and $1.16 \pm 0.05$, respectively), even though there were no significant differences in the carotid intima-media thickness. The maximum TBR values had the strongest positive correlation with hsCRP level among the various cardiovascular risk factors $(r=$ $0.68, P<0.01)$. However, other emerging inflammatory markers such as lipoprotein-associated phospholipase $A_{2}$ or monocyte chemoattractant protein-1 were not coherently associated with TBR values. Multiple stepwise regression analyses showed that hsCRP and diastolic blood pressure were independent decisive factors for maximum TBR, whereas age, diastolic blood pressure, and LDL-C were factors that determined the maximum intimamedia thickness. Conclusion: Vascular inflammation measured using ${ }^{18}$ F-FDG PET was increased in healthy individuals without hyperlipidemia but with elevated hsCRP.
\end{abstract}

Key Words: atherosclerosis; vascular inflammation; highsensitivity C-reactive protein; hyperlipidemia; ${ }^{18} \mathrm{~F}$-fluorodeoxyglucose positron emission tomography

J Nucl Med 2011; 52:10-17

DOI: 10.2967/jnumed.110.080838

\footnotetext{
Received Jul. 2, 2010; revision accepted Oct. 1, 2010.

For correspondence or reprints contact: Kyung Mook Choi, Division of Endocrinology and Metabolism, Department of Internal Medicine, Korea University, Guro Hospital, Korea University College of Medicine, Guro-dong, Guro-gu, Seoul 152-703, Korea.

E-mail: medica7@gmail.com

${ }^{*}$ Contributed equally to this work.

COPYRIGHT @ 2011 by the Society of Nuclear Medicine, Inc.
}

A therosclerosis is now recognized as an inflammatory disorder. The inflammatory state and composition of atherosclerotic plaques, rather than the degree of stenosis, are known as the core contributing factors responsible for acute cardiovascular events (1). However, angiography, the current gold standard imaging technique for atherosclerosis, is invasive and cannot provide information about plaque inflammation and vulnerability (2). Therefore, new imaging techniques that can demonstrate the dynamic biologic activity within atherosclerotic plaques are critically needed for further risk stratification and early prevention for acute cardiovascular events.

Recently, PET with ${ }^{18} \mathrm{~F}$-FDG has been suggested as a promising novel imaging technique to identify vascular inflammation (3). Several animal and human studies have shown that ${ }^{18} \mathrm{~F}$-FDG PET can visualize inflamed vessels, and ${ }^{18} \mathrm{~F}$-FDG uptake is strongly correlated with macrophage infiltration (4-7). In our previous study, the patients with impaired glucose tolerance or type 2 diabetes, compared with healthy subjects, had significantly increased maximum target-to-background ratio (TBR) values measured using ${ }^{18} \mathrm{~F}$ FDG PET (8). However, there have been no studies focusing on vascular inflammation of apparently healthy men and women stratified by low-density lipoprotein cholesterol (LDL-C) and high-sensitivity C-reactive protein (hsCRP) levels using ${ }^{18} \mathrm{~F}$-FDG PET.

Although LDL-C is the basis of current guidelines for risk stratification of cardiovascular disease (CVD), approximately $40 \%$ of deaths from CVD occur in patients with low cholesterol levels (9). Therefore, there has been growing interest in novel biomarkers that can provide information about the unexplained portion of cardiovascular risk in the conventional risk stratification system. hsCRP is a representative inflammatory biomarker that independently predicts future cardiovascular events and can enhance risk stratification, regardless of the LDL-C level (10). Along with hsCRP, lipoprotein-associated phospholipase $\mathrm{A}_{2}\left(\mathrm{Lp}-\mathrm{PLA}_{2}\right)$ and monocyte chemoattractant protein-1 (MCP-1) have been 
regarded as emerging biomarkers for the fine risk stratification of CVD $(11,12)$. However, there have been few studies to compare the relationship of various inflammatory biomarkers with vascular inflammation assessed by ${ }^{18} \mathrm{~F}-\mathrm{FDG}$ PET. Recently, Rudd et al. (13) demonstrated that arterial ${ }^{18} \mathrm{~F}-\mathrm{FDG}$ uptake showed the trends of positive correlation with levels of several circulating inflammatory biomarkers including hsCRP. In addition, Wu et al. (14) demonstrated that patients with higher ${ }^{18} \mathrm{~F}$-FDG uptake in the carotid artery had higher serum matrix metalloproteinase-1 levels. However, these previous 2 studies enrolled patients with known CVD not healthy subjects.

Therefore, in the current study we used ${ }^{18} \mathrm{~F}-\mathrm{FDG}$ PET to examine vascular inflammation of apparently healthy subjects stratified by LDL-C and hsCRP levels. Furthermore, we determined the correlation between circulating levels of emerging inflammatory markers, such as Lp-PLA $\mathrm{A}_{2}$ and MCP-1, and vascular inflammation measured using ${ }^{18} \mathrm{~F}-$ FDG PET. Last, we compared the factors that determine arterial wall inflammatory status, measured using ${ }^{18} \mathrm{~F}$-FDG PET, and carotid intima-media thickness (IMT) levels, measured by ultrasonography (which has been widely used to evaluate atherosclerosis burden, not composition).

\section{MATERIALS AND METHODS}

\section{Study Design and Subjects}

The participants were prospectively recruited from individuals who were self-referred for a routine health check-up at the Health Promotion Center of Korea, University Guro Hospital, between January and March 2009. Subjects were excluded from this study if they met any of the following criteria: history of CVD (myocardial infarction, unstable angina, stroke, or cardiovascular revascularization); diabetes; stage 2 hypertension (resting blood pressure, $\geq 160$ / $100 \mathrm{~mm} \mathrm{Hg}$ ); any lipid-lowering therapies and postmenopausal hormone replacement therapy for at least the 6-mo period before enrollment; history of inflammatory conditions that affect the study results, such as aortitis and vasculitis; taking medications that might affect inflammatory status, including steroid and nonsteroidal antiinflammatory drug within $6 \mathrm{mo}$; or malignancy or severe renal or hepatic disease. After ruling out subjects who met the exclusion criteria or who refused the study, we classified the remaining volunteers into the following 4 groups according to their baseline hsCRP and LDL-C levels, matching them by age as much as possible: subjects with low hsCRP and low LDL-C levels (hsCRP, $<2 \mathrm{mg} / \mathrm{L}$; LDL-C, $<130 \mathrm{mg} / \mathrm{dL}$ ), subjects with low hsCRP and high LDL-C levels (hsCRP, $<2 \mathrm{mg} / \mathrm{L}$; LDL-C, $\geq 130 \mathrm{mg} / \mathrm{dL}$ ), subjects with high hsCRP and low LDL-C levels (hsCRP, $\geq 2 \mathrm{mg} / \mathrm{L}$; LDL-C, $<130 \mathrm{mg} / \mathrm{dL}$ ), and subjects with high hsCRP and high LDL-C levels (hsCRP, $\geq 2 \mathrm{mg} / \mathrm{L} ; \mathrm{LDL}-\mathrm{C}, \geq 130 \mathrm{mg} / \mathrm{dL}$ ). As a result, 30 subjects in each of the 4 groups, for a total of 120 participants, were enrolled for this study. All participants provided written informed consent, and the Korea University Institutional Review Board, in accordance with the Declaration of Helsinki of the World Medical Association, approved this study protocol.

\section{Anthropometric and Laboratory Measurements}

The body mass index (BMI) was calculated as the weight/ height ${ }^{2}\left(\mathrm{~kg} / \mathrm{m}^{2}\right)$, and the waist circumference was measured at the midpoint between the lower border of the rib cage and the iliac crest. All blood samples were obtained in the morning after a 12-h overnight fast and were immediately stored at $-80^{\circ} \mathrm{C}$ for subsequent assays. Serum triglycerides and high-density lipoprotein cholesterol (HDL-C) were determined enzymatically using a chemistry analyzer (Hitachi 747; Hitachi Inc.). The LDL-C concentration was estimated using the Friedewald formula (15). A glucose oxidase method was used to measure plasma glucose. hsCRP levels were measured by Latex-enhanced Turbidimetric Immunoassay (HiSens hsCRP LTIA; HBI Co., Ltd.), with an interassay coefficient of variation of $7.2 \%$. Serum concentrations of Lp-PLA $\mathrm{A}_{2}$ were determined by an enzyme-linked immunosorbent assay kit (second-generation PLAC Test; diaDexus Inc.), with an interassay coefficient of variation between $6 \%$ and $7 \%$. The lower detection limit of Lp-PLA 2 in this assay was $2 \mathrm{ng} / \mathrm{mL}$. Serum MCP-1 levels were measured by enzyme-linked immunosorbent assay (R\&D Systems), with an interassay coefficient of variation between $4.6 \%$ and $6.7 \%$.

\section{Measurement of Carotid IMT}

The IMT of the common carotid artery was determined using high-resolution B-mode ultrasonography (EnVisor; Philips) with a 5- to $12-\mathrm{MHz}$ transducer. Measurements of carotid IMT were made using measurement software (Intimascope; Media Cross Co.) at 3 levels of the lateral and medial walls, $1-3 \mathrm{~cm}$ proximal to the carotid bifurcation. The mean IMT was the average value of 99 computer-based points in the region, and the maximal IMT was the IMT value at a maximal point of the region. All measurements were recorded by a single trained technician who was unaware of the subject's anthropometric and laboratory data.

\section{${ }^{18}$ F-FDG PET/CT}

PET/CT was performed using the Gemini TF 16-slice PET/CT scanner (Philips). The TF scanner is a new high-performance, timeof-flight-capable, fully 3-dimensional PET scanner using lutetium yttrium oxyorthosilicate crystals (16). After the patients had fasted at least $12 \mathrm{~h},{ }^{18} \mathrm{~F}-\mathrm{FDG}(5.19 \mathrm{MBq} / \mathrm{kg})$ was injected intravenously, and the patients rested in a quiet room for $60 \mathrm{~min}$. Whole-body PET images (below the cerebellum to the inguinal region) were acquired for $10 \mathrm{~min}$ ( $1 \mathrm{~min}$ per bed position). PET images were analyzed on a dedicated workstation (Extended Brilliance Workspace 3.5, with PET/CT viewer for automated image registration; Philips). The right carotid ${ }^{18} \mathrm{~F}$-FDG uptake was measured along the length of the right carotid vessel, starting at the bifurcation and extending inferiorly and superiorly every $4 \mathrm{~mm}$, for a total 10 consecutive PET/CT images for each subject. Arterial ${ }^{18}$ F-FDG uptake was quantified by a region of interest (ROI) around each artery on every slice of the coregistered transaxial PET/CT images. The ROI was fitted to the artery wall on each axial slice, and coronal and sagittal views were used to ensure that the ${ }^{18} \mathrm{~F}$-FDG uptake was from the artery. The standardized uptake value (SUV) is the decay-corrected tissue concentration of ${ }^{18} \mathrm{~F}$-FDG (in $\mathrm{kBq} / \mathrm{mL}$ ) divided by the injected dose per body weight $(\mathrm{kBq} / \mathrm{g})$. On each image slice, the mean and maximum SUVs of the ROI were measured as the mean and maximum pixel activity. The SUVs for all 10 slices within the right carotid artery were averaged to calculate the mean and maximums SUVs for each participant. Next, the arterial SUV was divided by the blood-pool SUV measured from the jugular vein (standardized circular ROIs; right carotid artery, area $=77.9 \pm 3.42 \mathrm{~mm}^{2}, 9$ pixels; and right jugular vein, area $=95.0 \pm 12.7 \mathrm{~mm}^{2}, 9$ pixels) for nor- 
malization; thereby, a mean and maximum value of TBR was acquired for each subject (17). To determine the variability of the mean and maximum TBR measurements, images from 20 subjects were analyzed twice several weeks apart by 2 readers who were unaware of the subjects' clinical history. The intra- and interobserver correlation coefficient of the mean and maximum TBR measurements were greater than 0.8 .

\section{Statistical Analysis}

Each variable was examined for a normal distribution. Data are expressed as the mean $\pm \mathrm{SD}$ or median (interquartile range, $25 \%-$ $75 \%$ ) or as a number (percentage). Differences among the groups were tested using ANOVA for normally distributed variables or the Kruskal-Wallis $\mathrm{H}$ test for skewed variables, and the subsequent comparisons were performed by Tukey's HSD post hoc analysis. Categoric variables were analyzed with the Pearson $\chi^{2}$ test. Differences of maximum TBR and maximum IMT values among the 4 groups were tested using an analysis of covariance after adjusting for age, sex, and BMI. Pearson correlation coefficients were calculated to evaluate the relationship between cardiovascular risk factors and mean/maximum TBR and cardiovascular risk factors and mean/maximum IMT. Before correlation analysis, a natural logarithmic transformation was performed for nonnormally distributed variables. The association between the TBR and each risk factor was assessed by dividing the patients into quartiles based on the maximum TBR, and the $P$ value for linear trend was calculated to determine the linear association of various cardiovascular risk factors with the increment of maximum TBR levels. Multiple linear stepwise regression analysis with maximum IMT or maximum TBR as a dependent variable was performed to identify the risk factors that determined the maximum IMT and maximum TBR. All statistical results were based on 2-sided tests. Data were analyzed using SPSS for Windows (version 12.0; SPSS Inc.). We regarded a $P$ value of less than 0.05 as statistically meaningful.

\section{RESULTS}

\section{Characteristics of Study Subjects Stratified by hsCRP and LDL-C Levels}

The clinical and biochemical characteristics of the study subjects are presented in Table 1. Although there were no significant differences in age or sex, the BMI, waist circumference, diastolic blood pressure, and triglyceride and fasting blood glucose levels were significantly different between the groups; specifically, these parameters increased with the increments of LDL-C and hsCRP levels (Table 1). However, systolic blood pressure, circulating MCP-1 levels, and HDL-C showed no significant difference between the 4 groups. The Lp-PLA 2 levels were significantly different between the lowhsCRP and low-LDL-C group and the other groups (Table 2). There was a sequential increment of maximum TBR levels based on the 4 groups, even after adjustment for age, sex, and BMI (Fig. 1). In particular, the maximum TBR levels of the high-hsCRP and low-LDL-C group were significantly higher than those in the low-hsCRP and low-LDL-C (1.28 \pm 0.03 vs. $1.14 \pm 0.03, P<0.01)$ or low-hsCRP and highLDL-C groups $(1.28 \pm 0.03$ vs. $1.17 \pm 0.03, P=0.02)$, although there were no significant differences in the maximum IMT levels between the groups (Fig. 1).

\section{Correlation Between Cardiovascular Risk Factors and Mean/Maximum TBR or Mean/Maximum IMT Levels}

Waist circumference, diastolic blood pressure, and LDL-C had a significant positive correlation with both mean/maximum TBR and mean/maximum IMT (Table 3). However, the BMI and triglyceride, fasting blood glucose, and hsCRP levels had a significant positive correlation only with the mean/maximum TBR, not with the mean/maximum IMT. In contrast,

TABLE 1

Baseline Characteristics of Study Subjects Stratified by hsCRP and LDL-C Levels

\begin{tabular}{|c|c|c|c|c|c|}
\hline \multirow[b]{3}{*}{ Characteristic } & \multicolumn{4}{|c|}{ hsCRP } & \multirow[b]{3}{*}{$P$} \\
\hline & \multicolumn{2}{|c|}{$<2 \mathrm{mg} / \mathrm{L}$} & \multicolumn{2}{|c|}{$\geq 2 \mathrm{mg} / \mathrm{L}$} & \\
\hline & $\begin{array}{c}\mathrm{LDL}-\mathrm{C}<130 \mathrm{mg} / \mathrm{dL} \\
(n=30)\end{array}$ & $\begin{array}{c}\mathrm{LDL}-\mathrm{C} \geq 130 \mathrm{mg} / \mathrm{dL} \\
(n=30)\end{array}$ & $\begin{array}{c}\mathrm{LDL}-\mathrm{C}<130 \mathrm{mg} / \mathrm{dL} \\
(n=30)\end{array}$ & $\begin{array}{c}\mathrm{LDL}-\mathrm{C} \geq 130 \mathrm{mg} / \mathrm{dL} \\
(n=30)\end{array}$ & \\
\hline Male $(n)$ & $16(53.3)$ & $18(60.0)$ & $14(46.7)$ & $22(73.3)$ & 0.19 \\
\hline Age (y) & $52.5 \pm 8.3$ & $49.7 \pm 8.3$ & $47.2 \pm 12.3$ & $51.2 \pm 10.2$ & 0.19 \\
\hline BMI $\left(\mathrm{kg} / \mathrm{m}^{2}\right)$ & $22.2 \pm 2.6^{\star}$ & $24.3 \pm 2.4^{\dagger}$ & $25.4 \pm 3.9^{\dagger}$ & $26.0 \pm 3.1^{\dagger}$ & $<0.01$ \\
\hline Waist circumference $(\mathrm{cm})$ & $78.7 \pm 7.6^{\star}$ & $84.1 \pm 7.3^{\dagger}$ & $86.0 \pm 7.9^{\dagger}$ & $89.1 \pm 6.4^{\dagger}$ & $<0.01$ \\
\hline Systolic blood pressure $(\mathrm{mm} \mathrm{Hg})$ & $121.4 \pm 14.6$ & $121.4 \pm 13.3$ & $125.0 \pm 13.8$ & $129.5 \pm 28.4$ & 0.29 \\
\hline Diastolic blood pressure $(\mathrm{mm} \mathrm{Hg})$ & $78.8 \pm 7.8^{\star}$ & $81.0 \pm 9.7^{\star}$ & $82.9 \pm 11.5^{\star}$ & $89.8 \pm 11.1^{\dagger}$ & $<0.01$ \\
\hline LDL-C (mg/dL) & $79.9 \pm 19.5^{\star}$ & $162.6 \pm 19.7^{\dagger}$ & $107.2 \pm 16.3^{\ddagger}$ & $156.2 \pm 23.4^{\dagger}$ & $<0.01$ \\
\hline HDL-C (mg/dL) & $47.1 \pm 15.9$ & $52.1 \pm 11.8$ & $46.8 \pm 12.9$ & $44.8 \pm 9.4$ & 0.15 \\
\hline Triglycerides $(\mathrm{mg} / \mathrm{dL})$ & $57.0(46.0-82.3)^{\star}$ & $102.0(72.8-148.8)^{\dagger}$ & $109.5(80.8-213.8)^{\dagger}, \neq$ & $142.0(112.3-190.8)^{\ddagger}$ & $<0.01$ \\
\hline Fasting blood glucose (mg/dL) & $72.9 \pm 11.2^{\star}$ & $92.0 \pm 10.2^{\dagger}$ & $95.5 \pm 13.2^{\dagger}$ & $98.9 \pm 10.2^{\dagger}$ & $<0.01$ \\
\hline hsCRP (mg/L) & $0.29(0.20-0.56)^{\star}$ & $0.52(0.41,0.73)^{\dagger}$ & $3.13(2.41,6.91)^{\ddagger}$ & $3.29(2.50,5.61)^{\ddagger}$ & $<0.01$ \\
\hline Current smoker $(n)$ & $6(20.0)$ & $11(36.7)$ & $7(24.1)$ & $9(30.0)$ & 0.50 \\
\hline
\end{tabular}

\footnotetext{
${ }^{\star}, t, \neq$ No statistical difference between 2 groups, based on Tukey’s HSD post hoc analysis.

Data are expressed as mean $\pm \mathrm{SD}$; median, with interquartile range in parentheses; or number, with percentage in parentheses. $P$ value for overall difference among groups was calculated from ANOVA, Kruskal-Wallis $\mathrm{H}$ test, or Pearson $\chi^{2}$ test.
} 
TABLE 2

Cardiovascular Biomarkers, IMT, and TBR Values of Study Subjects Stratified by hsCRP and LDL-C Level

\begin{tabular}{|c|c|c|c|c|c|}
\hline \multirow[b]{3}{*}{ Biomarker } & \multicolumn{4}{|c|}{ hsCRP } & \multirow[b]{3}{*}{$P$} \\
\hline & \multicolumn{2}{|c|}{$<2 \mathrm{mg} / \mathrm{L}$} & \multicolumn{2}{|c|}{$\geq 2 \mathrm{mg} / \mathrm{L}$} & \\
\hline & $\begin{array}{c}\mathrm{LDL}-\mathrm{C}<130 \mathrm{mg} / \mathrm{dL} \\
(n=30)\end{array}$ & $\begin{array}{c}\mathrm{LDL}-\mathrm{C} \geq 130 \mathrm{mg} / \mathrm{dL} \\
(n=30)\end{array}$ & $\begin{array}{c}\mathrm{LDL}-\mathrm{C}<130 \mathrm{mg} / \mathrm{dL} \\
(n=30)\end{array}$ & $\begin{array}{c}\mathrm{LDL}-\mathrm{C} \geq 130 \mathrm{mg} / \mathrm{dL} \\
(n=30)\end{array}$ & \\
\hline Lp-PLA ${ }_{2}(n g / m L)$ & $226.4 \pm 97.7^{*}$ & $138.2 \pm 43.4^{\dagger}$ & $160.4 \pm 67.0^{\dagger}$ & $161.9 \pm 52.0^{\dagger}$ & $<0.01$ \\
\hline MCP-1 (pg/mL) & $247.3 \pm 64.9$ & $301.0 \pm 152.2$ & $279.1 \pm 144.6$ & $314.9 \pm 143.1$ & 0.25 \\
\hline Mean IMT (mm) & $0.62 \pm 0.09^{\star}$ & $0.69 \pm 0.17^{\star}{ }^{\dagger}$ & $0.65 \pm 0.18^{\star}$ & $0.75 \pm 0.15^{\dagger}$ & 0.01 \\
\hline Maximum IMT (mm) & $0.74 \pm 0.12^{\star}$ & $0.83 \pm 0.20^{*},{ }^{\dagger}$ & $0.78 \pm 0.24^{*}$ & $0.92 \pm 0.20^{\dagger}$ & $<0.01$ \\
\hline Mean SUV & $1.57 \pm 0.22^{\star}$ & $1.54 \pm 0.21^{\star}$ & $1.62 \pm 0.20^{\star}{ }^{\dagger}$ & $1.72 \pm 0.26^{\dagger}$ & 0.01 \\
\hline Maximum SUV & $1.66 \pm 0.22^{*}$ & $1.67 \pm 0.24^{\star}$ & $1.75 \pm 0.21^{\star},^{\dagger}$ & $1.88 \pm 0.30^{\dagger}$ & $<0.01$ \\
\hline Mean TBR & $1.18 \pm 0.10^{\star}$ & $1.08 \pm 0.08^{\dagger}$ & $1.20 \pm 0.11^{*}$ & $1.46 \pm 0.22^{\ddagger}$ & $<0.01$ \\
\hline Maximum TBR & $1.12 \pm 0.10^{\star}$ & $1.16 \pm 0.05^{\star}$ & $1.29 \pm 0.13^{\dagger}$ & $1.61 \pm 0.22^{\ddagger}$ & $<0.01$ \\
\hline
\end{tabular}

age and systolic blood pressure had a significant positive relationship only with the mean/maximum IMT, not with the mean/maximum TBR. The mean/maximum TBR had the strongest positive correlation with hsCRP $(r=0.52$ and $r=0.68$, both $P<0.01$ ), although the mean/maximum IMT had no significant correlation with hsCRP levels (Fig. 2). Moreover, There was no significant correlation between maximum TBR and maximum IMT values $(r=0.11, P=$ 0.22). The serum MCP-1 levels had a significant positive correlation with maximum TBR but not with the mean TBR and mean/maximum IMT. The circulating Lp-PLA 2 levels had no significant correlation with the mean/maximum TBR and mean/maximum IMT.

\section{Cardiovascular Risk Factors Stratified by Quartiles of Maximum TBR Levels}

On the basis of increments of maximum TBR levels, the BMI; waist circumference; diastolic blood pressure; and triglyceride, fasting blood glucose, and hsCRP levels were increased (Supplemental Table 1; supplemental materials are available online only at http://jnm.snmjournals.org). However, the age, systolic blood pressure, and HDL-C and MCP-1 levels showed no significant trends with the increments of maximum TBR levels. Likewise, the mean/maximum IMT showed no significant increment after the rise in maximum TBR levels.

\section{Factors Determining Maximum IMT and Maximum TBR}

Multiple stepwise regression analyses showed that age $(P<0.01)$, diastolic blood pressure $(P<0.01)$, and LDL-C $(P=0.02)$ were significant risk factors determining maximum IMT $\left(R^{2}=0.39\right)$, whereas hsCRP $(P<0.01)$ and diastolic blood pressure $(P=0.04)$ were significant decisive risk factors for maximum $\operatorname{TBR}\left(R^{2}=0.22\right.$; Table 4$)$.
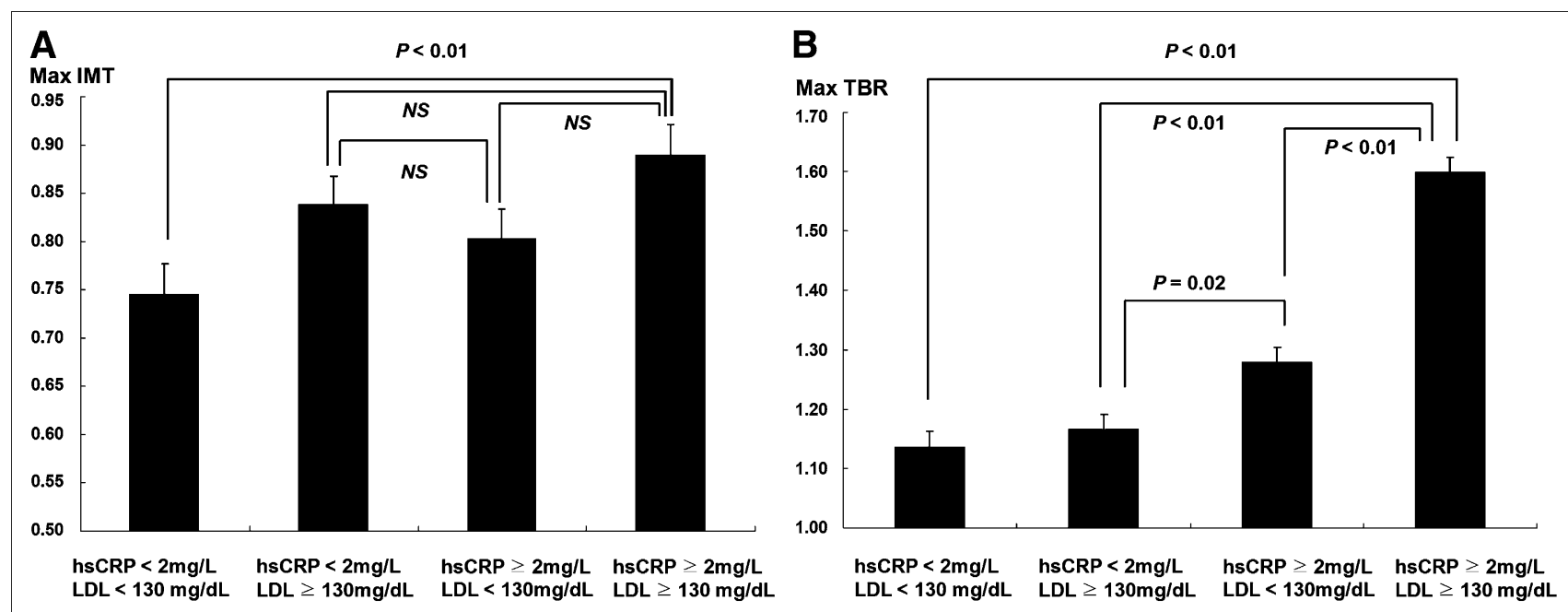

FIGURE 1. Maximum IMT (A) and maximum TBRs (B) by stratified groups according to hsCRP and LDL-C levels. $P$ value represents pairwise comparison based on Bonferroni's multiple-comparison procedure under analysis of covariance adjusted for age, sex, and BMI. Max = maximum; NS = nonsignificant. 
TABLE 3

Pearson Correlation Analysis Between Cardiovascular Risk Factors and Mean/Maximum TBR and IMT

\begin{tabular}{|c|c|c|c|c|c|c|c|c|}
\hline \multirow[b]{3}{*}{ Factor } & \multicolumn{4}{|c|}{ TBR } & \multicolumn{4}{|c|}{ IMT } \\
\hline & \multicolumn{2}{|c|}{ Mean } & \multicolumn{2}{|c|}{ Maximum } & \multicolumn{2}{|c|}{ Mean } & \multicolumn{2}{|c|}{ Maximum } \\
\hline & $r$ & $P$ & $r$ & $P$ & $r$ & $P$ & $r$ & $P$ \\
\hline Age & -0.01 & 0.95 & -0.05 & 0.60 & 0.52 & $<0.01$ & 0.49 & $<0.01$ \\
\hline BMI & 0.32 & $<0.01$ & 0.38 & $<0.01$ & 0.13 & 0.16 & 0.15 & 0.11 \\
\hline Waist circumference & 0.27 & $<0.01$ & 0.35 & $<0.01$ & 0.20 & 0.03 & 0.21 & 0.02 \\
\hline Systolic blood pressure & 0.14 & 0.13 & 0.13 & 0.15 & 0.23 & 0.01 & 0.3 & $<0.01$ \\
\hline Diastolic blood pressure & 0.31 & $<0.01$ & 0.32 & $<0.01$ & 0.29 & $<0.01$ & 0.33 & $<0.01$ \\
\hline LDL-C & 0.23 & 0.01 & 0.44 & $<0.01$ & 0.25 & 0.01 & 0.24 & 0.01 \\
\hline HDL-C & -0.13 & 0.17 & -0.08 & 0.36 & -0.05 & 0.57 & -0.07 & 0.47 \\
\hline Triglycerides* & 0.20 & 0.03 & 0.40 & $<0.01$ & 0.08 & 0.37 & 0.07 & 0.47 \\
\hline Fasting blood glucose & 0.22 & 0.01 & 0.42 & $<0.01$ & 0.14 & 0.11 & 0.16 & 0.09 \\
\hline hsCRP* & 0.52 & $<0.01$ & 0.68 & $<0.01$ & 0.14 & 0.12 & 0.15 & 0.11 \\
\hline Lp-PLA 2 & 0.06 & 0.63 & -0.14 & 0.16 & -0.07 & 0.47 & -0.07 & 0.45 \\
\hline MCP-1 & 0.13 & 0.20 & 0.24 & 0.01 & 0.00 & 0.98 & -0.05 & 0.65 \\
\hline
\end{tabular}

*Logarithmic transformed data were used.

\section{DISCUSSION}

The present study showed a steady increase in maximum TBR levels according to groups stratified by LDL-C and hsCRP levels. Compared with the low-hsCRP and low-LDL-C group or the low-hsCRP and high-LDL-C group, the high-hsCRP and low-LDL-C group had increased vascular inflammation, measured using ${ }^{18} \mathrm{~F}$-FDG PET. Furthermore, hsCRP levels and diastolic blood pressure were independent factors determining maximum TBR levels measured by ${ }^{18} \mathrm{~F}-$ FDG PET, whereas age, diastolic blood pressure, and LDL-C levels were important decisive factors for maximum IMT values assessed using ultrasonography. However, circulating Lp-PLA $A_{2}$ and MCP-1 levels were not coherently associated with TBR or IMT values in the study subjects.

Inflammation is a crucial element in the atherosclerotic process and contributes to all of its stages, from plaque initiation to growth and rupture (18). However, current imaging techniques for atherosclerotic burden, such as coronary angiography or carotid IMT, assess the severity of luminal stenosis but provide no information about plaque inflamma-
FIGURE 2. Correlation curves between hsCRP and mean/maximum TBR (A and B) and mean/maximum IMT for all the participants (C and D). After logarithmic transformation of hsCRP, Pearson correlation coefficients and corresponding $P$ values are displayed.

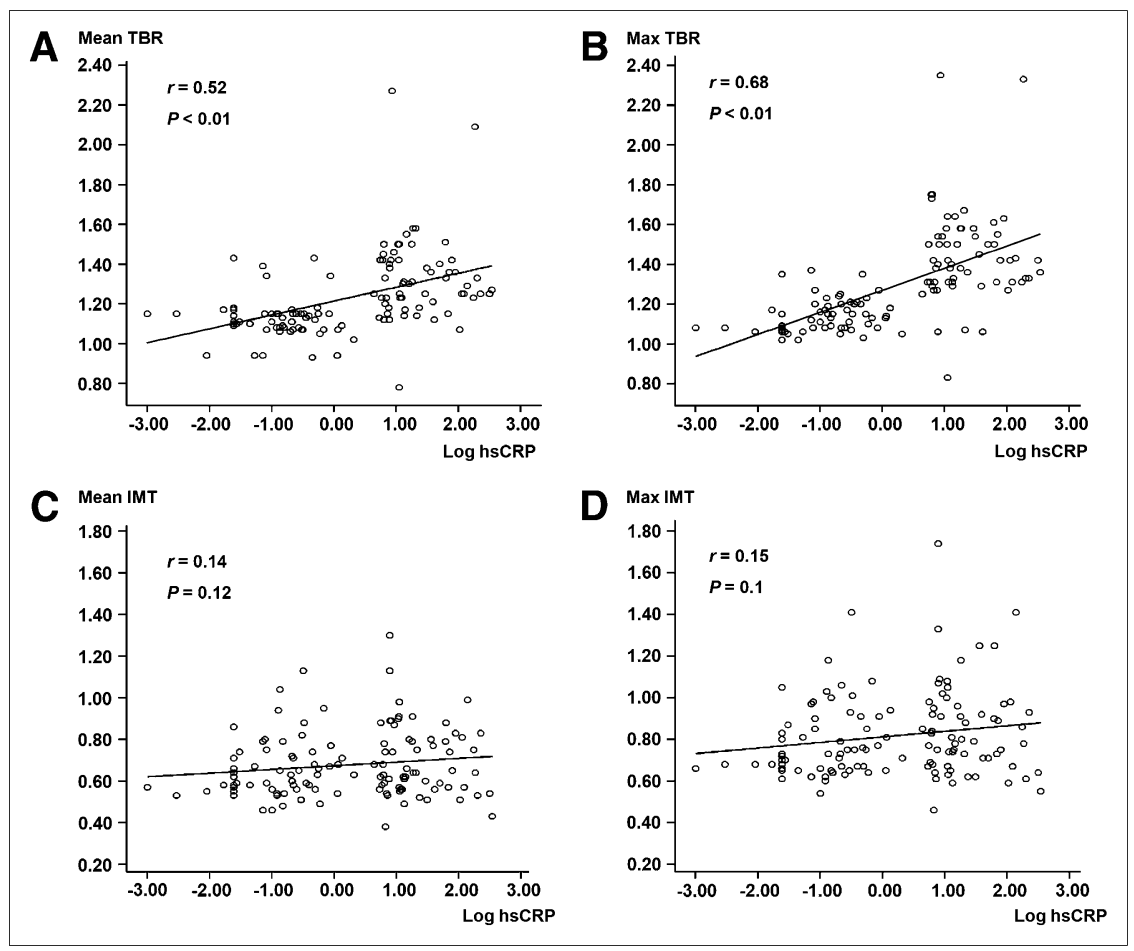


TABLE 4

Multiple Stepwise Regression Analyses for Determinant Factors Associated with Maximum IMT or Maximum TBR

\begin{tabular}{lcccc}
\hline Dependant variable & B & SE & $P$ & $R^{2}$ \\
\hline Maximum IMT & & & & \\
Age & $9.0 \times 10^{-3}$ & 0.001 & $<0.01$ & \\
Diastolic blood pressure & $4.5 \times 10^{-3}$ & 0.001 & $<0.01$ & \\
LDL-C & $8.9 \times 10^{-4}$ & 0.000 & 0.02 & 0.39 \\
Maximum TBR & & & & \\
$\quad$ hsCRP & $2.5 \times 10^{-2}$ & 0.006 & $<0.01$ & \\
Diastolic blood pressure & $3.1 \times 10^{-3}$ & 0.001 & 0.04 & 0.22
\end{tabular}

$\mathrm{B}=$ regression coefficient; $R^{2}=$ coefficient of determination. Independent variables in multiple stepwise regression analysis are sex, age, BMI, smoking history, systolic blood pressure, diastolic blood pressure, fasting blood glucose, LDL-C, HDL-C, triglycerides, hsCRP, Lp-PLA ${ }_{2}$, and MCP-1.

tion and vulnerability. Recent animal and clinical studies have shown that only ${ }^{18} \mathrm{~F}$-FDG PET would be a useful tool for the measurement of vascular inflammation. Tawakol et al. (19) reported a significant correlation between ${ }^{18} \mathrm{~F}-\mathrm{FDG}$ uptake from carotid plaques and macrophage staining from the corresponding histologic sections obtained by endarterectomy. The present study showed that vascular inflammation measured using ${ }^{18} \mathrm{~F}-\mathrm{FDG}$ PET was associated with various cardiovascular risk factors. Especially, the serum hsCRP levels had the strongest positive correlation with TBR values.

A large number of CVD events occur in asymptomatic patients, with risk defined as intermediate using current global risk assessment tools (20); however, conventional risk factors predict only $60 \%-65 \%$ of cardiac risk (21). Therefore, improving the predictability of cardiovascular events by a new diagnostic tool is an important focus for effective primary prevention. Consequently, several inflammatory markers have received much attention as an emerging screening method to predict cardiovascular events. Recent metaanalyses have indicated that adding hsCRP to risk assessment among persons at intermediate risk improves CVD risk stratification (22). According to the Atherosclerosis Risk In Communities study, individuals with an LDL-C level less than 130 $\mathrm{mg} / \mathrm{dL}$ and an hsCRP level more than $3.0 \mathrm{mg} / \mathrm{L}$, compared with those with an hsCRP level less than $1.0 \mathrm{mg} / \mathrm{L}$ in an adjusted model, had an increased coronary heart disease risk (hazard ratio, 1.76; 95\% confidence interval, 1.02-3.03) (23). Furthermore, Ridker et al. (24) reported that the relative risk of a first cardiovascular event for women in the highest quintile of hsCRP was 2.34 (1.6-3.4, $P<0.001)$, compared with the lowest, whereas the corresponding relative risk in the highest quintiles of LDL-C was $1.5(1.1-2.0, P<0.001)$. These data suggest that hsCRP, as a predictor of cardiovascular events, might be a comparable to or even stronger than LDL-C level. In agreement with those studies, our results showed that the maximum TBR levels of the high-hsCRP and low-LDL-C group were significantly higher than those of the low-hsCRP and high-LDL-C group (1.29 \pm 0.13 vs. $1.16 \pm 0.05, P<0.01)$. Moreover, the hsCRP levels had a strong correlation with the maximum TBR, reflecting vascular inflammation, whereas the LDL-C level had an independent relationship with the carotid IMT, implying an atherosclerotic burden. Therefore, considering that hsCRP and LDL-C may identify different aspects of cardiovascular risk, screening for both circulating biomarkers might provide better prognostic information than screening for either alone.

Recently, the JUPITER study (25) demonstrated that rosuvastatin significantly reduces incident CVD by $44 \%$ and all-cause mortality by approximately $20 \%$ in apparently healthy people without hyperlipidemia but with elevated hsCRP levels. This finding suggests that elevated hsCRP levels might indicate future risk of CVD in healthy individuals who are not currently candidates for statin treatment. The current study showed increased vascular inflammation measured using ${ }^{18} \mathrm{~F}-\mathrm{FDG}$ PET in individuals with the same criteria used in the JUPITER study (LDL-C $<130$ $\mathrm{mg} / \mathrm{dL}$ and hsCRP $\geq 2 \mathrm{mg} / \mathrm{L}$ ), compared with individuals with decreased LDL-C and hsCRP levels, even though the IMT values were not different. Several previous studies have also reported that circulating hsCRP levels have been shown to correlate poorly with results of tests that quantify the extent of atherosclerosis, such as carotid IMT (26) and CT for coronary calcium (27). These results suggest that vascular inflammation detected by ${ }^{18}$ F-FDG PET might reflect dynamic changes of atherosclerosis at an earlier stage than the atherosclerotic burden detected by ultrasonography or CT. Furthermore, ${ }^{18}$ F-FDG PET might be a useful noninvasive imaging tool to predict response to medical treatment. Tahara et al. (28) showed that ${ }^{18}$ F-FDG uptake of atherosclerotic plaque was significantly decreased in simvastatin-treated humans after only 3 mo of treatment. In that study, the decrease in the ${ }^{18} \mathrm{~F}-\mathrm{FDG}$ uptake was not correlated with LDL-C reduction, suggesting the antiinflammatory effect of statin might be independent of a LDL-C lowering effect. These results intimate the possibility that ${ }^{18}$ F-FDG PET might be useful to evaluate the drug effects on acute cardiovascular events caused by inflammatory atherosclerotic plaque before more expensive largescale clinical trials.

Along with hsCRP, Lp-PLA 2 and MCP-1 have been suggested to be useful biomarkers for predicting atherosclerotic CVD. Lp-PLA $A_{2}$ is an enzyme mainly produced by macrophages and generates bioactive proatherogenic lipids, contributing to monocyte recruitment and increased expression of endothelial adhesion molecules (29). Because of its vascular specificity, Lp-PLA 2 is an especially appealing biomarker. Koenig et al. (30) reported that the plasma concentration of Lp-PLA 2 was strongly associated with cardiovascular events after controlling for traditional risk factors. MCP-1, a member of the CC chemokine family, is known to be involved in the pathogenesis of atherosclerosis by promoting recruitment of inflammatory cells to the vessel wall (31). Hoogeveen et al. (32) showed a significant association of MCP-1 levels with 
peripheral artery disease, independent of other CVD risk factors. However, we did not find that circulating Lp-PLA and MCP-1 levels had a clear relationship with vascular inflammation measured using ${ }^{18} \mathrm{~F}-\mathrm{FDG}$ PET. In agreement with our results, many previous studies examining the prognostic utility of circulating Lp-PLA ${ }_{2}$ have yielded conflicting results depending on sex (33), cholesterol levels (23), and lipid-lowering therapy (34). In contrast, circulating hsCRP levels as a biomarker are stable over a long time without diurnal variation (35) and can be measured inexpensively using standardized methods for clinical use.

There were some limitations to our study. Because of the drawback of a cross-sectional design, we could not clarify the causal relationship between LDL-C, inflammatory biomarkers, and vascular inflammation measured using ${ }^{18} \mathrm{~F}-\mathrm{FDG}$ PET. Therefore, we are exploring the longitudinal effect of various kinds of lipid-lowering medications on ${ }^{18} \mathrm{~F}-\mathrm{FDG}$ uptake along with changes in several inflammatory markers, including hsCRP. However, the present study has several strengths, such as study design, including recruitment plan using predefined inclusion and exclusion criteria, compared with several previous retrospective studies that enrolled patients who underwent ${ }^{18} \mathrm{~F}$-FDG PET studies for cancer follow-up care.

\section{CONCLUSION}

Compared with individuals with low hsCRP and high LDL-C levels (hsCRP $<2 \mathrm{mg} / \mathrm{L}$ and LDL-C $\geq 130 \mathrm{mg} / \mathrm{dL}$ ) or low hsCRP and low LDL-C levels (hsCRP $<2 \mathrm{mg} / \mathrm{L}$ and LDL-C $<130 \mathrm{mg} / \mathrm{dL}$ ), healthy subjects with high hsCRP and low LDL-C levels (hsCRP $\geq 2 \mathrm{mg} / \mathrm{L}$ and LDL-C $<130 \mathrm{mg} / \mathrm{dL}$ ) had increased vascular inflammation, measured using ${ }^{18}$ F-FDG PET. However, no significant differences in IMT were observed. TBR levels measured using ${ }^{18} \mathrm{~F}-\mathrm{FDG}$ PET are independently associated with hsCRP levels and diastolic blood pressure, whereas IMT values are related to age, diastolic blood pressure, and LDL-C levels. Further studies are needed to determine the clinical roles of ${ }^{18} \mathrm{~F}-\mathrm{FDG}$ PET and hsCRP in prognostic and therapeutic implications for cardiovascular events.

\section{ACKNOWLEDGMENTS}

This study was supported by the Korea Science and Engineering Foundation (KOSEF) funded by the Korean government (R01-2007-000-20546-0) and a grant from the Korean Health 21 R\&D Project, Ministry of Health and Welfare, Republic of Korea (A 050463). The funding sources had no role in the study design. The financial supporters had no control over the collection, analyses, or interpretation of any study data, nor did they control the writing of the article or the decision to submit the manuscript for publication.

\section{REFERENCES}

1. van Lennep JE, Westerveld HT, van Lennep HW, Zwinderman AH, Erkelens DW, van der Wall EE. Apolipoprotein concentrations during treatment and recurrent coronary artery disease events. Arterioscler Thromb Vasc Biol. 2000;20:2408-2413.
2. Pasterkamp G, Schoneveld AH, van der Wal AC, et al. Relation of arterial geometry to luminal narrowing and histologic markers for plaque vulnerability: the remodeling paradox. J Am Coll Cardiol. 1998;32:655-662.

3. Chen W, Bural GG, Torigian DA, Rader DJ, Alavi A. Emerging role of FDG$\mathrm{PET} / \mathrm{CT}$ in assessing atherosclerosis in large arteries. Eur J Nucl Med Mol Imaging. 2009;36:144-151.

4. Ogawa M, Ishino S, Mukai T, et al. ${ }^{18} \mathrm{~F}-\mathrm{FDG}$ accumulation in atherosclerotic plaques: immunohistochemical and PET imaging study. J Nucl Med. 2004;45: 1245-1250.

5. Tawakol A, Migrino RQ, Hoffmann U, et al. Noninvasive in vivo measurement of vascular inflammation with F-18 fluorodeoxyglucose positron emission tomography. J Nucl Cardiol. 2005;12:294-301.

6. Zhang Z, Machac J, Helft G, et al. Non-invasive imaging of atherosclerotic plaque macrophage in a rabbit model with F-18 FDG PET: a histopathological correlation. BMC Nucl Med. 2006;6:3.

7. Rudd JH, Warburton EA, Fryer TD, et al. Imaging atherosclerotic plaque inflammation with $\left[{ }^{18} \mathrm{~F}\right]$-fluorodeoxyglucose positron emission tomography. Circulation. 2002;105:2708-2711.

8. Kim TN, Kim S, Yang SJ, et al. Vascular inflammation in patients with impaired glucose tolerance and type 2 diabetes: analysis with ${ }^{18} \mathrm{~F}$-fluorodeoxyglucose positron emission tomography. Circ Cardiovasc Imaging. 2010;3:142-148.

9. Smith SC Jr.. Current and future directions of cardiovascular risk prediction. Am J Cardiol. 2006;97(suppl 1):28-32.

10. Kones R. The Jupiter study, CRP screening, and aggressive statin therapy: implications for the primary prevention of cardiovascular disease. Ther $A d v$ Cardiovasc Dis. 2009;3:309-315.

11. Anderson JL. Lipoprotein-associated phospholipase A2: an independent predictor of coronary artery disease events in primary and secondary prevention. Am J Cardiol. 2008;101(suppl):S23-S33.

12. Coll B, Alonso-Villaverde C, Joven J. Monocyte chemoattractant protein-1 and atherosclerosis: is there room for an additional biomarker? Clin Chim Acta. 2007;383:21-29.

13. Rudd JH, Myers KS, Bansilal S, et al. Relationships among regional arterial inflammation, calcification, risk factors, and biomarkers: a prospective fluorodeoxyglucose positron-emission tomography/computed tomography imaging study. Circ Cardiovasc Imaging. 2009;2:107-115.

14. Wu YW, Kao HL, Chen MF, et al. Characterization of plaques using ${ }^{18}$ F-FDG $\mathrm{PET} / \mathrm{CT}$ in patients with carotid atherosclerosis and correlation with matrix metalloproteinase-1. J Nucl Med. 2007;48:227-233.

15. Friedewald WT, Levy RI, Fredrickson DS. Estimation of the concentration of low-density lipoprotein cholesterol in plasma, without use of the preparative ultracentrifuge. Clin Chem. 1972;18:499-502.

16. Surti S, Kuhn A, Werner ME, Perkins AE, Kolthammer J, Karp JS. Performance of Philips Gemini TF PET/CT scanner with special consideration for its time-offlight imaging capabilities. J Nucl Med. 2007;48:471-480.

17. Rudd JH, Myers KS, Bansilal S, et al. Atherosclerosis inflammation imaging with ${ }^{18}$ F-FDG PET: carotid, iliac, and femoral uptake reproducibility, quantification methods, and recommendations. J Nucl Med. 2008;49:871-878.

18. Hansson GK. Inflammation, atherosclerosis, and coronary artery disease. $N$ Engl J Med. 2005;352:1685-1695.

19. Tawakol A, Migrino RQ, Bashian GG, et al. In vivo ${ }^{18} \mathrm{~F}$-fluorodeoxyglucose positron emission tomography imaging provides a noninvasive measure of carotid plaque inflammation in patients. J Am Coll Cardiol. 2006;48:18181824.

20. Ford ES, Giles WH, Mokdad AH. The distribution of 10-year risk for coronary heart disease among US adults: findings from the National Health and Nutrition Examination Survey III. J Am Coll Cardiol. 2004;43:1791-1796.

21. Baldassarre D, Amato M, Pustina L, et al. Measurement of carotid artery intima-media thickness in dyslipidemic patients increases the power of traditional risk factors to predict cardiovascular events. Atherosclerosis. 2007;191: 403-408.

22. Buckley DI, Fu R, Freeman M, Rogers K, Helfand M. C-reactive protein as a risk factor for coronary heart disease: a systematic review and meta-analyses for the U.S. Preventive Services Task Force. Ann Intern Med. 2009;151:483-495.

23. Ballantyne CM, Hoogeveen RC, Bang H, et al. Lipoprotein-associated phospholipase A2, high-sensitivity C-reactive protein, and risk for incident coronary heart disease in middle-aged men and women in the Atherosclerosis Risk in Communities (ARIC) study. Circulation. 2004;109:837-842.

24. Ridker PM, Rifai N, Rose L, Buring JE, Cook NR. Comparison of C-reactive protein and low-density lipoprotein cholesterol levels in the prediction of first cardiovascular events. N Engl J Med. 2002;347:1557-1565.

25. Ridker PM, Danielson E, Fonseca FA, et al. Rosuvastatin to prevent vascular events in men and women with elevated C-reactive protein. $N$ Engl J Med. 2008;359: 2195-2207. 
26. Folsom AR, Pankow JS, Tracy RP, et al. Association of C-reactive protein with markers of prevalent atherosclerotic disease. Am J Cardiol. 2001;88:112-117.

27. Redberg RF, Rifai N, Gee L, Ridker PM. Lack of association of C-reactive protein and coronary calcium by electron beam computed tomography in postmenopausal women: implications for coronary artery disease screening. J Am Coll Cardiol. 2000;36:39-43.

28. Tahara N, Kai H, Ishibashi M, et al. Simvastatin attenuates plaque inflammation: evaluation by fluorodeoxyglucose positron emission tomography. J Am Coll Cardiol. 2006;48:1825-1831.

29. MacPhee CH, Moores KE, Boyd HF, et al. Lipoprotein-associated phospholipase A2, platelet-activating factor acetylhydrolase, generates two bioactive products during the oxidation of low-density lipoprotein: use of a novel inhibitor. Biochem J. 1999;338:479-487.

30. Koenig W, Twardella D, Brenner H, Rothenbacher D. Lipoprotein-associated phospholipase A2 predicts future cardiovascular events in patients with coronary heart disease independently of traditional risk factors, markers of inflammation, renal function, and hemodynamic stress. Arterioscler Thromb Vasc Biol. 2006;26:1586-1593.
31. Rossi D, Zlotnik A. The biology of chemokines and their receptors. Annu Rev Immunol. 2000;18:217-242.

32. Hoogeveen RC, Morrison A, Boerwinkle E, et al. Plasma MCP-1 level and risk for peripheral arterial disease and incident coronary heart disease: Atherosclerosis Risk in Communities study. Atherosclerosis. 2005;183:301-307.

33. Packard CJ, O'Reilly DS, Caslake MJ, et al. Lipoprotein-associated phospholipase A2 as an independent predictor of coronary heart disease. West of Scotland Coronary Prevention Study Group. N Engl J Med. 2000;343:11481155 .

34. O'Donoghue M, Morrow DA, Sabatine MS, et al. Lipoprotein-associated phospholipase A2 and its association with cardiovascular outcomes in patients with acute coronary syndromes in the PROVE IT-TIMI 22 (PRavastatin Or atorVastatin Evaluation and Infection Therapy-Thrombolysis In Myocardial Infarction) trial. Circulation. 2006;113:1745-1752.

35. Ockene IS, Matthews CE, Rifai N, Ridker PM, Reed G, Stanek E. Variability and classification accuracy of serial high-sensitivity $\mathrm{C}$-reactive protein measurements in healthy adults. Clin Chem. 2001;47:444-450. 